\title{
Linx
}

Revue des linguistes de l'université Paris X Nanterre

$12 \mid 2002$

«Comme la lettre dit la vie »

\section{Je, me moi : allomorphes ou facettes différentes de la première personne?}

Danielle Leeman

\section{(2) OpenEdition}

\section{Journals}

Édition électronique

URL : http://journals.openedition.org/linx/1290

DOI : $10.4000 / \operatorname{linx} .1290$

ISSN : 2118-9692

\section{Éditeur}

Presses universitaires de Paris Nanterre

\section{Édition imprimée}

Date de publication : 1 octobre 2002

Pagination : 118-124

ISSN : 0246-8743

\section{Référence électronique}

Danielle Leeman, « Je, me moi : allomorphes ou facettes différentes de la première personne ? », Linx

[En ligne], 12 | 2002, mis en ligne le 10 octobre 2012, consulté le 19 avril 2019. URL : http:// journals.openedition.org/linx/1290; DOI : 10.4000/linx.1290

Ce document a été généré automatiquement le 19 avril 2019

Département de Sciences du langage, Université Paris Ouest 


\title{
Je, me moi : allomorphes ou facettes différentes de la première personne?
}

\author{
Danielle Leeman
}

« Mangez-moi » dit la gaufrette

(M. Perret, $1988: 32$ )

Le plus souvent, les formes pronominales sont présentées comme les variantes combinatoires d'un même morphème ; ainsi, je, me, moi seraient les trois concrétisations de l'unité "première personne", susceptible de se réaliser différemment selon sa fonction. Toutefois, si l'on admet que le sens ne se réduit pas à la référence, en constatant que, quoique désignant la même personne, le vainqueur d'Austerlitz et le vaincu de Waterloo n'aboutissent pas à une affirmation tautologique dans

Le vainqueur d'Austerlitz est le vaincu de Waterloo

on est amené de même à envisager l'hypothèse que je, me, moi n'ont pas la même identité sémantique en observant que

Je suis moi

Je voudrais être moi

Je ne suis plus moi-même

ne forment pas non plus des énoncés inconsistants, circulaires ou contradictoires. De plus, on ne peut pas relier de manière bi-univoque une forme à une fonction puisque, si je semble effectivement dévolu uniquement à la position de sujet, en revanche moi et peutêtre me peuvent également avoir cette fonction :

\begin{tabular}{|l|l|l|l|}
\hline & $(1)$ & a & Moi, Danielle Leeman, atteste avoir reçu de M. Max Dupont la somme de..., le... \\
\hline & & b & Eve me fait rire ${ }^{1}$ \\
\hline
\end{tabular}

et d'autre part me et moi peuvent aussi bien l'un que l'autre être compléments d'objet direct ou indirect : 


\begin{tabular}{|l|l|l|l|}
\hline & $(2)$ & a & Il me regarde/Regarde-moi \\
\hline & & b & Il me sourit/Il pense à moi \\
\hline & & c & Il m'a photographié/ « Pourquoi soupçonnes-tu cet homme parce qu'il a pris quelques \\
\hline & & & photos ? - Il a surtout photographié moi à ce meeting, c'est cela qui m'inquiète » \\
\hline & & & (M. Ronat, $1979: 118)$. \\
\hline
\end{tabular}

Si je, me, moi peuvent tous trois être sujets, et si me, moi peuvent tous deux être objets, ils ne sont pas interchangeables ${ }^{2}$ :je ne commute ni avec moi ni avec me en (1), me et moi ne sont pas substituables en (2). Il y a donc en fait une répartition des trois formes selon la construction ; mais, contrairement à l'affirmation d'E. Benveniste (op. cit.) «Cela n'arrive jamais, on le sait », il existe des cas de neutralisation ${ }^{3}$ :

\begin{tabular}{|l|l|l|l|}
\hline & $(3)$ & $a$ & Il ne m'a pas prêté attention/Il n'a pas prêté attention à moi \\
\hline & & $b$ & Cela m'est inhérent/Cela est inhérent à moi \\
\hline
\end{tabular}

2 Cet ensemble de remarques (cf. aussi D. Leeman 2000 et 2002) conduit à l'hypothèse que je , me, moi ne sont pas de simples allomorphes. Les trois formes ont certes le même sens si l'on entend par là qu'elles réfèrent à la personne qui parle (définition habituelle des grammaires), mais si l'on s'en tient là, on ne peut pas expliquer les contraintes qui règlent leur emploi (d'ailleurs «je et tu peuvent aussi être a-référentiels », M. Perret, 1994 : 49) : outre son sens référentiel (qui la rapproche des deux autres), chaque forme a une identité propre, que l'on va essayer d'atteindre en interprétant les constructions dans lesquelles elle apparaît. Du même coup est postulé que les variantes morphosyntaxiquement libres $(3 . a, b)$ ne sont en fait pas exactement synonymes.

\section{Je distingué de me et moi}

3 La forme je ne connaît que la position sujet mais avec la contrainte que le pronom n'est pas séparé du verbe - sauf éventuellement par un autre clitique et dans la formule je, soussigné. La forme me ne peut être sujet que d'un infinitif subordonné. La forme moi apparaît avec l'infinitif de narration, qui, lui, entre dans une proposition indépendante (Et moi de rire) et dans les cas de coordination (4.a), emphase (4.b), modification par un adjectif (4.c), ellipse du groupe verbal (4.d) :

\begin{tabular}{|l|l|l|l|}
\hline & $(4)$ & $a$ & Eve et moi sommes amoureuses de Max \\
\hline & & $b$ & C'est moi qui l'ai fait \\
\hline & & $c$ & Moi seul te comprends \\
\hline
\end{tabular}




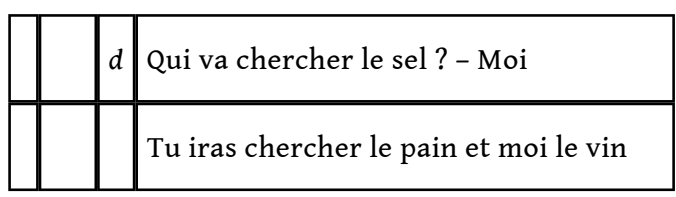

Pour résumer, je est sujet seulement d'un verbe conjugué (par opposition à me et moi), il nécessite la présence d'un verbe mais n'admet aucune modification (par opposition à moi ) : il est le seul sujet possible.

4 Si l'on connaissait le sens de cette position, on aurait sans doute la possibilité de préciser le statut de je, qui ne peut occuper que cette fonction. L'interprétation qui lui est traditionnellement affectée est que le sujet désigne celui qui fait l'action (l'agent) ou qui est dans un certain état (le patient) - définition quasi contradictoire! Mais on ne peut admettre qu'il s'agisse là de la caractérisation de la position elle-même : en fait, on décrit le statut attribué à son sujet par un verbe particulier (cf. S. Meleuc, 2000) - malgré la démonstration par C. Fillmore de la non-coïncidence entre « cas » et fonction. La question est de savoir ce qui est commun à Paul en (5.a) et (5.b), attendu que dans ces deux cas Paul sera dit « sujet » :

\begin{tabular}{|l|l|l|l|}
\hline & $(5)$ & $a$ & Paul abat un arbre (il agit) \\
\hline & & $b$ & Paul est malade (il est dans un état) \\
\hline
\end{tabular}

Admettons à la suite de S. C. Dik (1997 : 248) que le sens de la position est que la situation est présentée du point de vue du N qui l'occupe : Paul ; si l'on compare $(6 . a, b)$ :

\begin{tabular}{|l|l|l|l|}
\hline & (6) & $a$ & Paul a abattu un arbre \\
\hline & & $b$ & Un arbre a été abattu par Paul \\
\hline
\end{tabular}

le point commun entre Paul et un arbre est que l'événement est peint du point de vue de Paul en (6.a) et du point de vue de l'arbre en (6.b). Puisque je ne peut être que sujet, et étant donné l'hypothèse d'interprétation que l'on a retenue pour cette fonction, on dispose de bases nouvelles pour caractériser je : je réfère à la personne qui parle en tant qu'elle énonce un point de vue; par opposition à me/moi (lorsqu'ils sont sujets), ce point de vue a les propriétés suivantes :

- il concerne un événement inscrit dans le temps (je est nécessairement le sujet d'un verbe conjugué);

- la personne qui parle est exhibée en tant que seul auteur possible de ce discours ( je exclut d'être associé à un autre sujet, que ce soit explicitement - par coordination - ou implicitement - par emphase);

- la personne qui parle est montrée comme étroitement dépendante de ce qu'elle rapporte (je ne peut apparaître sans verbe ou groupe verbal). Ainsi que l'a déjà signalé N. Landais (cf. D. Leeman, 2001a), je parle de ce qui suit mais réciproquement l'énoncé qui le suit parle de je.

Dans ces conditions, je s'oppose à me en ce que, me n'étant jamais le sujet du verbe conjugué, il ne peut véhiculer le point de vue auquel le fait est asserté ; en (1.b), me énonce, en tant que sujet de l'infinitif, le point de vue qui guide l'interprétation de 
l'infinitif, mais c'est un point de vue nécessairement subordonné, enchâssé dans le cadre défini par le sujet du verbe conjugué. Ce qui distingue je et moi, c'est que moi sujet énonce le point de vue de la personne qui parle d'une part en tant qu'elle est associée à d'autres personnes $(4 . a, b, c)$ et d'autre part en tant qu'elle est autonome par rapport au fait rapporté (4.d).

\section{Me opposé à je et moi}

7 Si me s'oppose à je lorsqu'il est susceptible d'être sujet ainsi qu'on l'a dit ci-dessus, il s'en distingue surtout en ce qu'il est principalement complément, fonction que ne peut assurer je. Autrement dit, si je est définissable comme porteur du point de vue auquel est asserté l'énoncé, me est essentiellement l'objet du propos, donc présente la première personne comme ce dont il est question dans la phrase. Ce rôle sémantique ne se confond pas avec la définition traditionnelle du complément d'objet, qui en fait un " patient » (ce qui subit l'action exprimée par le verbe), sens qui peut aussi bien être celui du sujet :

(7) Le représentant palestinien achoppe sur cette clause 4

8 En tant que complément, me s'oppose à moi de la même façon que, en tant que sujet, je s'oppose à moi : clitique, me ne peut être coordonné, ni séparé du verbe ni faire l'objet d'une emphase ou d'une restriction. On avancera donc pour me la même hypothèse que pour je: me présente la personne qui parle en tant qu'elle constitue l'objet du discours (tandis que je la montre comme source du point de vue auquel il est énoncé) mais, par opposition à moi, me exhibe la première personne comme le seul objet de ce discours et comme entièrement dépendante du procès rapporté ${ }^{5}$. En revanche, la forme moi suppose une situation par rapport à d'autres individus, explicite (8.a, b) ou implicite (8.c, d) :

\begin{tabular}{|l|l|l|l|}
\hline & (8) & $a$ & Max me parle/Max parle à moi et à personne d'autre \\
\hline \hline & & $b$ & Eve a vu Luc et moi \\
\hline & & $c$ & Ali ne voit que moi \\
\hline & & $d$ & C'est moi qu'elle vise en disant cela \\
\hline
\end{tabular}

et, séparable du verbe, elle est capable d'un emploi autonome, donc du point de vue sémantique se définit indépendamment du procès véhiculé par le verbe. Ces déductions sont compatibles avec l'hypothèse de G. Kleiber $(1994: 128)$ que les formes toniques ont trait à l'ostension : « le geste semble en effet inconvenant avec les formes clitiques :

? Tu partiras avec Jean (+ geste pour désigner l'interlocuteur)

?Je suis d'accord (+ geste du locuteur qui se désigne soi-même)

alors qu'il apparaît compatible avec les formes accentuées :

Toi (+ geste sur une personne qui sera l'interlocuteur), tu partiras avec Jean

Moi, je suis d'accord (+ geste du locuteur qui se désigne soi-même). »

Et que l'ostension - compatible en cela avec l'accent que reçoit la forme tonique « accomplit une identification autonome de l'objet désigné » (ibid:133). 

expliquer pourquoi on a moi à l'impératif positif (Regarde-moi) mais me à l'impératif négatif (Ne me regarde pas), si l'on admet que la forme négative présuppose la forme positive (O. Ducrot, 1984 : 215 sqq.) : l'énoncé en ne pas se construit sur une relation ayant déjà défini l'objet (me) par le procès qui le concerne (D. Leeman, 2001a). Dans le même ordre d'idées, Donne-m'en ou Fais-m'y penser s'expliquent par le fait qu'une première étape a posé moi : Donne-moi de cela ou Fais-moi penser à cela-d'où le $m e(m$ ) dans la deuxième étape (qui présuppose la première) : Donne-m'en, Fais-m'y penser.

l'ensemble des comportements syntaxiques de moi observés (rappelons que la forme tonique équivaut à un nom), on peut tirer l'hypothèse que ce pronom donne une identité « lexicale » de la personne, c'est-à-dire à la manière dont un nom le ferait (D. Leeman, 2001c) : en la présentant en tant qu'elle est dotée d'un ensemble de propriétés spécifiques qui la distingue des autres. Cette définition permet de justifier que ce soit moi et non me qui apparaît en position attributive: disant Je suis moi, on assume et revendique une personnalité particulière qui ne saurait se ramener au lot commun ; souhaitant Je voudrais être moi, on prend acte d'une dissociation entre ce que l'on est et la plénitude non réalisée de sa véritable nature; déplorant Je ne suis plus moi (-même) on constate à nouveau cette non-coïncidence avec l'être profond définitoire de la personne.

\section{Me et moi dans la complémentation des adjectifs}

11 Partant de l'idée - répandue, on l'a vu - que « clitiques objets directs et clitiques objets indirects sont les uns et les autres en distribution complémentaire avec les NP complets, et par suite aussi avec les formes fortes, semblables aux NP» (1977 : 74-75), R. Kayne remarque "la non-homogénéité des datifs " à propos des verbes (par exemple Elle lui sourit vs Elle pense à lui) et des adjectifs (Jean lui est semblable vs Jean est pareil à lui) mais sans lui trouver d'explication; en admettant l'existence d'un trait [+ datif] permettant la cliticisation de à $N$ (que posséderaient sourire, semblable, mais non penser, pareil), il reste à «montrer que l'attribution du trait [- datif] aux compléments en à en français est toujours, ou presque toujours, prédictible à partir de leur valeur sémantique, c'est-à-dire qu'il y a un contenu sémantique dans la notion de "datif" » (ibid. : 147) - mais l'auteur n'a en la matière pas d'hypothèse à avancer.

12 Partant de l'idée, contraire à la sienne, que clitiques et formes fortes ont une identité sémantique propre, et munie d'une hypothèse quant à la teneur de cette signification, on se donne pour objectif de voir s'il y aurait une explication possible à leur répartition dans la complémentation adjectivale, en relation avec le sens de l'adjectif ; le corpus est celui qu'a rassemblé et classé L. Picabia (1978), plus particulièrement les tables mettant en jeu un complément en à, les autres prépositions supposant toujours le pronom disjoint - ce qu'il serait intéressant d'expliquer!

\section{III.1. Table 1 (p. 114)}

On constate qu'un adjectif construit avec à $\mathrm{N}$ suppose, en ce qui concerne la pronominalisation, trois comportements : ou bien le complément est disjoint (à moi), ou bien le complément est conjoint $(m e)$, ou bien les deux sont possibles mais au prix d'un 
changement de sens (sensible), ce dernier cas justifiant que l'on examine si la répartition $\grave{a}$ moi/me n'est pas corrélable à une raison sémantique :

\begin{tabular}{|c|c|c|}
\hline$(9)$ & $a$ & Paul est abonné à moi/* Paul m'est abonné \\
\hline & $b$ & Paul est allergique à moi/* Paul m'est allergique \\
\hline & $c$ & Paul est attentif à moi/* Paul m'est attentif \\
\hline & $d$ & * Paul est favorable à moi/Paul m'est favorable \\
\hline & $e$ & * Paul est hostile à moi/Paul m'est hostile \\
\hline & $f$ & * L'heure est propice à moi/L'heure m'est propice \\
\hline & $g$ & Paul est réfractaire à moi/* Paul m'est réfractaire \\
\hline & $h$ & 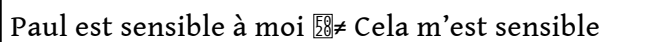 \\
\hline
\end{tabular}

La comparaison des propriétés syntaxiques (telles que testées par L. Picabia) ne donne en effet pas de résultat probant: propice se distingue de tous les autres du côté des sujets, admettant un $\mathrm{N}$ [-hum] et non un $\mathrm{N}$ [+hum], mais du point de vue qui nous intéresse il se range avec hostile et favorable qui, eux, demandent uniquement un sujet [+hum $]^{6}$. Du côté des compléments, tous ont en commun de refuser une complétive à l'indicatif (Paul est abonné à ce que je vienne/*viens prendre le thé tous les jours), l'effacement de àce au profit du seul que, la construction en de la part (Paul est attentif à me faire plaisir/*Il est attentif de la part de Paul de me faire plaisir) ou de permettre la pronominalisation de la complétive par $y$ (Paul y est abonné/attentif). Pour les autres propriétés, ils ne s'opposent pas de manière systématique : ainsi, propice refuse l'emploi absolu, mais c'est aussi le cas de abonné (dans le cadre testé, évidemment), or le premier se construit avec me et le second avec à moi.

On partira donc de l'hypothèse que la différence de construction à moi/me attire l'attention sur le fait que les adjectifs supposant tel même type de complément doivent avoir quelque chose en commun du point de vue du sens - et symétriquement s'opposer à ceux qui réclament tel autre type de complément. On se bornera ici à quelques remarques informelles, de façon à simplement voir si l'hypothèse aurait des chances d'être vérifiée par un travail plus approfondi.

Si l'on en croit les définitions lexicographiques, allergique, réfractaire et hostile, qui ont trait à une attitude négative, s'opposent par le fait que les deux premiers supposent une expérimentation concrète entrainant une réaction de rejet de la part du sujet, tandis que hostile décrit une attitude globale a priori: être allergique au sport, c'est ne pas en supporter l'exercice ; être réfractaire au sport, c'est refuser de le pratiquer; être hostile au sport, ce peut simplement être opposé à l'idée d'en faire ou que les autres en fassent. Notons que hostile est susceptible d'avoir un sujet non humain, donc non doté d'intentionnalité.

La comparaison de attentif avec favorable et propice aboutit au même commentaire; les trois concernent une attitude positive mais se distinguent par une différence d'engagement de la part du sujet : être attentif, c'est "porter attention à », « agir avec 
application ", "veiller avec soin ", tandis que favorable (qui admet un sujet non humain) et propice caractérisent une « disposition » a priori bienveillante.

Les deux séries ont donc ceci en commun que les adjectifs construits avec à moi présentent la relation établie entre le sujet et l'objet comme une réaction du sujet à l'existence de l'objet, tandis que les adjectifs construits avec me la montrent comme une propriété partant du sujet - ou interprétée comme telle, dans le cas où le sujet est non humain : Les vents nous sont favorables/propices - et s'appliquant à l'objet. On peut de la sorte opposer :

\begin{tabular}{|l|l|l|l|}
\hline & $(10)$ & $a$ & Paul est sensible à moi \\
\hline \hline & & $b$ & L'urgence de la situation me devient sensible \\
\hline
\end{tabular}

L'énoncé (10.a) décrit l'effet que ma présence, mes actions, mes paroles font sur Paul (le sujet). L'énoncé (10.b) décrit l'effet que l'urgence de la situation fait sur me (l'objet). Ces conclusions sont compatibles avec les hypothèses avancées pour l'identité des deux formes pronominales: dans le premier cas, moi désigne la personne conçue indépendamment de ce que véhicule le prédicat et susceptible d'être distinguée des autres; dans le second, me la montre concernée par l'événement rapporté sans opposition par rapport à autrui ${ }^{7}$ :

\begin{tabular}{|l|l|l|l|}
\hline & $(11)$ & $a$ & Paul est sensible à moi et non à toi \\
\hline & & $b$ & ?? L'urgence de la situation me devient sensible et non à toi \\
\hline
\end{tabular}

\section{III.2. Table 5 (p. 118)}

Cette classe se définit (L. Picabia, op. cit.) par la concurrence possible de à et pour: Il est facile à/pour Paul de faire cela. Je ne retiens que les phrases les plus naturelles (selon moi); par exemple, je dirais moins facilement ? Cela m'est bienfaisant que Cela m'est bénéfique, ?Cela m'est capital que Cela m'est important, ?Cela m'est dur que Cela m'est difficile (par opposition à Cela est bienfaisant/capital/dur pour moi). L'ensemble des adjectifs se construit avec le clitique (12.a) ; indifférent admet aussi le pronom disjoint mais la phrase change alors de sens (12.b); particulier et propre admettent les deux constructions sans modification du rôle des actants (12.c) $)^{8}$ :

\begin{tabular}{|l|l|l|l|}
\hline & $(12)$ & a & $\begin{array}{l}\text { Cela m'est agréable, bénéfique, commode, contraire, coutumier, difficile, } \\
\text { douloureux, }\end{array}$ \\
\hline & & & pénible, utile, vital... \\
\hline & & $b$ & Paul m'est indifférent $\neq$ Paul est indifférent à moi \\
\hline & & c & Cela m'est particulier/Cela est particulier à moi \\
\hline
\end{tabular}


Cela m'est propre/Cela est propre à moi

Le cas (12.a) se ramène au commentaire de (10.b) ci-dessus. En (12.b), Paul m'est indifférent décrit également l'effet produit par le sujet (Paul) sur l'objet (me), tandis qu'à l'inverse, Paul est indifférent à moi rend compte de l'effet produit par l'objet (moi) sur le sujet (Paul) - ce qui permet de rapprocher cet énoncé de (10.a). La convergence de ces observations permet d'avancer une hypothèse sur ce qui peut distinguer les deux types de formulation en (12.c) : Cela m'est particulier juge une relation entre cela et me telle qu'elle part de cela pour s'appliquer à me; il est dit de cela qu'il s'agit d'une particularité qui a trait à ma personne. En revanche Cela est particulier à moi subordonne le statut de cela à celui de moi pris comme repère : c'est son rapport à moi qui permet de caractériser cela comme une particularité.

\section{III.3. Confirmations}

Dans la table 6 (p. 120), seuls aimable et gentil se construisent avec à $N$ [hum]; la pronominalisation par le clitique est impossible :

\begin{tabular}{|l|l|l|l|}
\hline & $(13)$ & $a$ & Cela est aimable/gentil à toi \\
\hline & & $b$ & $*$ Cela t'est aimable/gentil \\
\hline
\end{tabular}

Ce qui est jugé aimable ou gentil, c'est le fait que tu aies fait cela; les deux énoncés réagissent à une action particulière de toi, exhibé comme la source justifiant le qualificatif attribué à cela. C'est également moi le repère du jugement porté sur le sujet Paul dans (table 7, p. 121) :

(14) a Paul est analogue/équivalent/pareil/semblable à moi

Cette classe comporte aussi l'adjectif égal, susceptible des deux types de complément mais avec un changement de sens :

(14) $b$ Cela m'est égal/Paul est égal à moi

Le premier énoncé enregistre un jugement sur les faits produit par cela sur l'objet ( $m e$ ) tandis que dans le second, moi est l'aune à laquelle on évalue le sujet (Paul) : comme pour les autres exemples, statuer sur Paul (le sujet) présuppose que l'on ait statué sur moi (l'objet), doté par conséquent d'une autonomie par rapport à l'état du sujet, lui étant extérieur. A nouveau, donc, l'hypothèse avancée pour distinguer les deux formulations de (3.b) ${ }^{9}$ rejoint ce que l'on a dit de (12.c) : Cela m'est inhérent définit l'objet (me) par son rapport au sujet (cela) tandis que Cela est inhérent à moi fait de moi la référence de la caractérisation de cela. Ce rôle d'« étalon» de moi se retrouve dans la table 8 (p. 122) avec : 
(15) a Léa est parallèle/perpendiculaire/tangente à moi

et dans la table 12 (p. 127) pour :

(15) $b$ Cela est antérieur/postérieur/ultérieur à moi

par opposition par exemple à :

(15)

Max m'est antipathique/cher/étranger/fidèle/sympathique...

où l'adjectif décrit l'effet produit par le sujet (Max) sur l'objet (me). Et l'on n'assimilera pas les formulations (16) à d'exacts synonymes, conformément à ce qui a été dit à propos de (3.b) et (12.c) :

\begin{tabular}{|l|l|l|l|}
\hline$(16)$ & $a$ & Paul est inférieur à moi/Paul m'est inférieur \\
\hline & & $b$ & Paul est supérieur à moi/Paul m'est supérieur \\
\hline
\end{tabular}

\section{Conclusion provisoire} nouvelle : J. Damourette et E. Pichon la soutiennent déjà de même que G. Guillaume, dans la première moitié $d u 20^{e}$ siècle ( $c f$. D. Leeman, 2001c). Elle revient sur le devant de la scène avec certains linguistes cognitivistes, tels L. Talmy (1988: 69sqq.) ou G. Lakoff (1996: 91sqq. $)^{10}$, qui corrèlent les allomorphes chacun à une certaine "partie» de la personne : je joue le rôle de la raison et me celui du désir dans Je me retiens d'être impoli (L. Talmy), de même je est la conscience et me l'être qui agit concrètement dans Je me déteste d'avoir fait cela (G. Lakoff).

La démarche ici adoptée consiste à tâcher de dépasser l'intuition référentielle ou conceptuelle qui, trop dépendante de ce qu'évoquent les relations lexicales, se heurte rapidement à des contre-exemples : ainsi que l'a pointé C. Fillmore (op. cit.), je et me ont le même statut dans Je reçois un coup et On me donne un coup, et en revanche les deux je ne correspondent pas aux mêmes rôles de la personne dans Je reçois un coup et Je donne un coup. Dire que je, en tant que forme dévolue à la position sujet, présente la personne en tant qu'auteur du point de vue auquel est asserté l'énoncé paraît au contraire valoir pour l'ensemble des exemples ici considérés.

Partir des formes permet également de s'apercevoir que n'est pas exacte l'affirmation selon laquelle on aurait affaire à une distribution complémentaire stricte entre me et moi - quand bien même elle le serait, elle n'explique pas pourquoi certains compléments sont conjoints et d'autres sont disjoints. On rencontre en fait quatre configurations, puisque, outre les deux possibilités mutuellement exclusives précédentes, existent le cas où les deux sont possibles pour des emplois différents (Max m'est indifférent/Max est indifférent à 
moi) et celui où les deux sont possibles sans modification actancielle (Max m'est supérieur/ Max est supérieur à moi). L'identité de chaque forme ne peut donc être trouvée dans ce qu'elle désigne mais plutôt dans la manière dont elle présente ce qu'elle désigne, ce que confirme une brève étude de la complémentation des adjectifs : il resterait à vérifier si l'hypothèse avancée à propos de à «non datif» (selon l'expression de R. Kayne, op. cit.) peut être étendue aux autres prépositions, qui supposent nécessairement le pronom disjoint.

\section{BIBLIOGRAPHIE}

\section{Références}

ARMENGAUD, F. et al. (1984) La ronde des sujets, DRLAV 30, Centre de Recherche de l'Université de Paris VIII.

BENVENISTE, E. (1965 in 1974) «L'antonyme et le pronom en français moderne », Problèmes de linguistique générale II, Paris, Gallimard.

DAMOURETTE, J. et PICHON, E. (1933 et 1943) Des mots à la pensée. Essai de grammaire de la langue française, tomes III et IV, Paris, D'Artrey.

DIK, S. C. $\left(1997^{2}\right)$ The Theory of Functional Grammar, I, Berlin-New York, Mouton de Gruyter.

DUBOIS, J. et al. (1979) Dictionnaire de la langue française Lexis, Paris, Larousse.

DUCROT, O. (1984), Le dire et le dit, Paris, Minuit.

FILLMORE, C. (1965, republ. 1969) « Toward a Modern Theory of Case », in D. Reibel et S. Schane (eds) Modern Studies in English, Prentice Hall, Englewoods Cliffs.

GUILLAUME, G. (1988 et 1989), Leçons de linguistique de Gustave Guillaume, 1947-1948C et 1946-1947C, tomes VIII et IX, Presses universitaires de Lille et Presses de l'université Laval, Québec.

GUIMIER, E. (1996 in 1998) « Les constructions à prédicat de l'objet des verbes de la perception », in M. Forsgreen, K. Jonasson et H. Kronning (eds), Prédication, Assertion, Information, Uppsala, Acta Universitatis Upsaliensis.

KAYNE, R. (1975 trad. 1977) Syntaxe du français. Le cycle transformationnel, Paris, Le Seuil.

KLEIBER, G. (1992 in 1994) «Y a-t-il un il ostensif? », Anaphores et pronoms, Louvain-la-Neuve, Duculot.

LAKOFF, G. (1996) « Sorry, I'm not myself today: The Metaphor System for Conceptualizing the Self », in G. Fauconnier \& E. Sweetser (eds), Spaces, Worlds and Grammar, Chicago \& London, The University of Chicago Press.

LEEMAN, D. (2000 in 2001a) « Le pronom personnel et ses variantes », colloque Grammaire et Grammaires organisé par le C.I.E.P. à l'Ecole Polytechnique, 9-10 octobre, Paris, Didier.

LEEMAN, D. (2000 in 2001b) « La polysémie du pronom personnel », colloque La Polysémie organisé par 0 . Soutet à la Sorbonne, 17-18 novembre, Presses de la Sorbonne. 
LEEMAN, D. (2002) « Me est un autre », Hommages offerts à Michel Arrivé, Paris, L'Harmattan.

MELEUC, S. (2000) « Pour un traitement lexical du verbe », Le français aujourd'hui 131, Paris, AFEF.

NØLKE, H. (1997) « Anaphoricité et focalisation : le cas du pronom personnel disjoint », in W. de Mulder, L. Tasmowski-De Ryck, C. Vetters (eds), Relations anaphoriques et (in)cohérence, Amsterdam-Atlanta, Rodopi.

PERRET, M. (1988) Le signe et la mention. Adverbes embrayeurs ci, ça, la, ilvec en moyen français, Genève-Paris, Droz.

PERRET, M. (1994) L'énonciation en grammaire du texte, Paris, Nathan-université, coll. 128.

PICABIA, L. (1978) Les constructions adjectivales en français, Genève-Paris, Droz.

RONAT, M. (1979) «Pronoms topiques et pronoms distinctifs », Langue française 44, Paris, Larousse.

TALMY, L. (1988) « Force Dynamics in Language and Cognition », Cognitive Science 12.

\section{NOTES}

1. Le statut de me dans ce type de structure est controversé ; au moins sémantiquement, le pronom a une relation privilégiée avec l'infinitif, qui conditionne son acceptabilité avec le verbe introducteur (E. Guimier, 1996 in 1998).

2. D'où le jugement sévère d'E. Benveniste (1974 : 198) à l'encontre de J. Damourette et E. Pichon.

3. On ne peut donc pas non plus souscrire au «principe » énoncé par H. Nølke (1997:60) : «Les pronoms disjoints (toniques) s'emploient exactement là où les conjoints ne sont pas permis ».

4. Selon le Lexis, achopper se définit : "être arrêté par une difficulté, un obstacle »; le sujet de ce verbe a donc un rôle de patient.

5. Cette dépendance est telle que, dans le cas de certains verbes pronominaux, on ne voit pas quelle est l'identité propre de me: Je m'absente demain, Je m'aperçois d'une erreur. On n'en conclura pas pour autant qu'il s'agit d'un mot «vide»: il y a bien une différence entre, par exemple, Je m'aperçois d'une erreur et J'aperçois une erreur.

6. Les adjectifs de la table 1 sont rassemblés sur le critère qu'ils admettent la construction à ce que $P$ : Max est favorable à ce que Luc vienne.

7. Comme clitique, me ne peut évidemment pas être spécifié par des adverbes tels que uniquement , seulement : Paul est sensible à moi uniquement/seulement.

8. J'admets plus facilement Ça, c'est particulier à moi ! ou Ça, c'est propre à moi ! que * C'est agréable à moi!, * C'est bénéfique à moi!, * C'est commode à moi!, etc.

9. L'adjectif inhérent fait partie de la table 7 (L. Picabia, op. cit.).

10. Cf. D. Leeman (2000-2001b) pour une présentation de ces propositions et une évaluation de leur éventuelle adéquation pour le français. 


\section{AUTEUR}

\section{DANIELLE LEEMAN}

Université de Paris 10 - Nanterre U.M.R. 7114 (MOdèles, DYnamique des langues, COrpus) 